九州大学学術情報リポジトリ

Kyushu University Institutional Repository

\title{
3D Finite Difference Time Domain Simulation of Microwave Propagation in a Coaxial Cable
}

Yousefian, Ali

Graduate School of Engineering Sciences, Kyushu University

Yamamoto, Naoji

Faculty of Engineering Sciences, Kyushu University

https://doi.org/10.5109/1957495

出版情報 : Evergreen. 5 (3)，pp.1-11，2018-09. 九州大学グリーンアジア国際リーダー教育センター バージョン：

権利関係 : 


\title{
3D Finite Difference Time Domain Simulation of Microwave Propagation in a Coaxial Cable
}

\author{
Ali Yousefian*, Naoji Yamamoto \\ Graduate School of Engineering Sciences, Kyushu University, Japan \\ *Author to whom correspondence should be addressed, \\ E-mail: 3ES17308W@s.kyushu-u.ac.jp
}

(Received December 4, 2017; accepted July 31, 2018).

We developed a three dimensional FDTD (Finite Difference Time Domain) approximation to Maxwell's equations simulation for propagation of TEM (Transverse Electro-Magnetic) microwaves in a coaxial cable. The model consists of a standard coaxial cable using a 3D Cartesian coordinate system with a microwave input of $10 \mathrm{Ws}$ and a frequency of $24 \mathrm{GHz}$. This simulation considers a realistic input of microwave electromagnetic fields utilizing a plane format in the cable and the results are compared to the conventional four point input method. Furthermore the challenges of converting a cylindrical cable to a Cartesian grid system are discussed and their influence on the accuracy of the simulation is analyzed.

Keywords: FDTD, Numerical Simulation, Electric Propulsion, Microwave.

\begin{tabular}{|c|c|}
\hline \multicolumn{2}{|c|}{ Nomenclature } \\
\hline$\Delta t$ & : Time step \\
\hline$\Delta x, \Delta y, \Delta z$ & : Grid spacing \\
\hline$\varepsilon_{d i}$ & : Relative permittivity of dielectric region \\
\hline$n z$ & : Simulation grid number in $\mathrm{z}$ direction \\
\hline$E_{x}$ & : Electric field in $x$ direction \\
\hline$E_{y}$ & : Electric field in $y$ direction \\
\hline$E_{z}$ & : Electric field in $z$ direction \\
\hline$E_{r}$ & : Electric field in radial direction \\
\hline$B_{x}$ & : Magnetic field in $\mathrm{x}$ direction \\
\hline$B_{y}$ & : Magnetic field in y direction \\
\hline$B_{z}$ & : Magnetic field in z direction \\
\hline$B_{\vartheta}$ & : Magnetic field in rotational direction \\
\hline$\varepsilon$ & : Permittivity \\
\hline$\mu$ & : Permeability \\
\hline$i, j, k$ & : Simulation spatial index \\
\hline$n$ & : Simulation time index \\
\hline$r_{\text {outer }}$ & : Coaxial cable’s outer radius \\
\hline$r_{\text {inner }}$ & : Coaxial cable’s inner radius \\
\hline$\omega$ & : Microwave’s angular frequency \\
\hline$v_{0}$ & : Voltage within the cable \\
\hline$\varnothing$ & : $\quad$ Phase difference \\
\hline
\end{tabular}

$\begin{array}{lll}C_{D} & : & \text { Speed of light in dielectric } \\ r & : & \text { Radial distance } \\ P_{\text {in }} & : & \text { Input power } \\ P_{\text {out }} & : & \text { Power output } \\ U_{\text {field }} & : & \text { Total field energy } \\ V & : & \text { Simulation cell volume }\end{array}$

\section{Introduction}

FDTD (Finite Difference Time Domain) simulation method is extensively used in plasma simulation as a way to calculate the fluctuations of electric and magnetic field values with respect to space and time. Therefore, this method is utilized to simulate propagation of

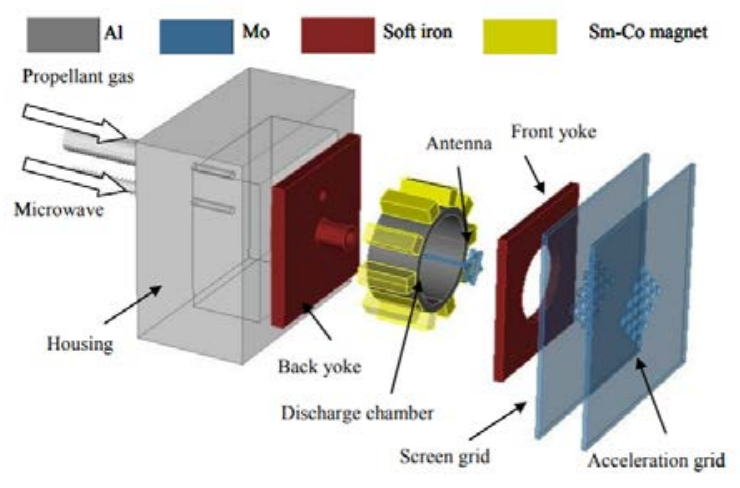

Fig. 1: An ion thruster developed in Yamamoto lab in Kyushu University2) 
electromagnetic waves and charged particles. Our research focuses on simulation of particles inside a miniature ion thruster. An ion engine works by creating plasma through ECR (Electron Cyclotron Resonance) method and accelerating the resultant ions out of the engine thus producing thrust. Ion thrusters have already been used extensively in space missions, such as Deep Space 1, HAYABUSA, and other missions. Miniature ion engines are candidates for use as miniature propulsion system for small satellites. The adoption of ion engines for small satellites will lead to the expansion of satellite's capability. That is, missions such as Mars exploration and self-disposal of satellites would be possible.

It is, however, difficult to measure inner properties of the plasma, since the miniature ion engine is so small that we cannot insert measurement equipment without perturbations. Numerical simulations are effective tool to understand the phenomena occurring in a miniature microwave ion thruster ${ }^{1)}$. Coaxial cables are used in order to feed the microwave power to the engine and an accurate simulation of microwave propagation inside a coaxial cable leads to a more realistic simulation of particles for the entire engine. Figure 1 shows one such engine developed in our research group in Kyushu University with the coaxial cable attached to the thruster.

Previous works in this area focuses on developing a 3D coupling PIC (Particle in Cell) - FDTD code for analyzing the plasma behavior in ion thruster ${ }^{3)}$. The microwave input method used in the previous works involves a simplistic coaxial cable with a partial microwave input ${ }^{4-9)}$. This method which is also known as four point method is discussed further in section 4. In this paper first, we will analyze a more comprehensive microwave simulation in an actual coaxial cable geometry elaborated in section 2 with a comprehensive microwave input method in 3D model. This input method being briefly introduced in textbook $^{10)}$ was also inspired by references 11 and 12 in which a two dimensional cable was simulated. There are no published works which focuses on the details of microwave input for a 3D model in detail in this field hence the motivation for our work. Then the results of our comprehensive simulation are validated and finally our method is compared with the conventional four point method and their accuracy is analyzed.

\section{Simulation Geometry}

The three dimensional simulation model considers the actual cable used in Figure 1 (Huber+Suhner Type EZ_141_TP_M17_COIL) to feed the microwaves into the thruster. The general outline of the cable and simulation grids are illustrated in Figure 2. The cable has a length of $50 \mathrm{~mm}$ and an outer diameter of $3.58 \mathrm{~mm}$. A time step of $d t=2 \times 10^{-14} s$ and a grid size of $d x=d y=d z=$ $10^{-4} \mathrm{~m}$ and are adopted for this 3D model which leads to the grid numbers illustrated in Figure 2. The cable has a capacitance of $98 \mathrm{pf} / \mathrm{m}$ which leads to a relative permittivity of $\varepsilon_{d i}=2.178 \varepsilon_{0}$ at the dielectric region.

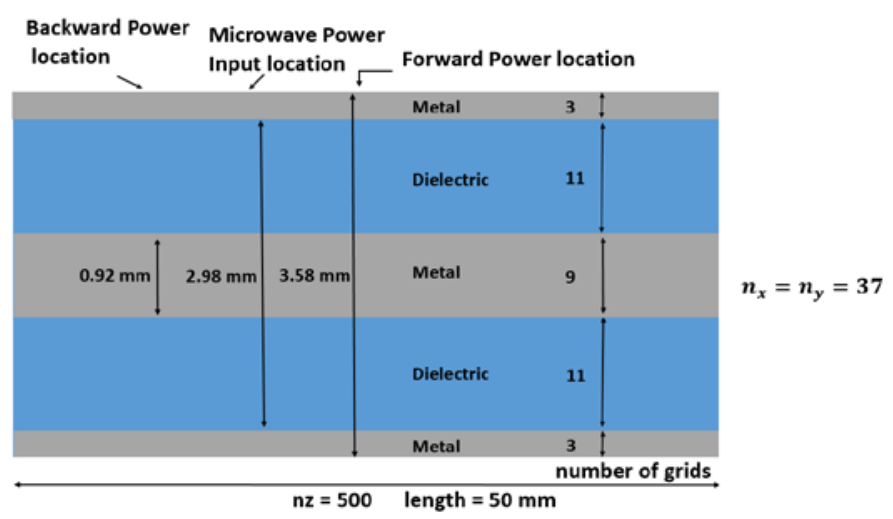

Fig. 2: Cross section of the cable in the simulation model

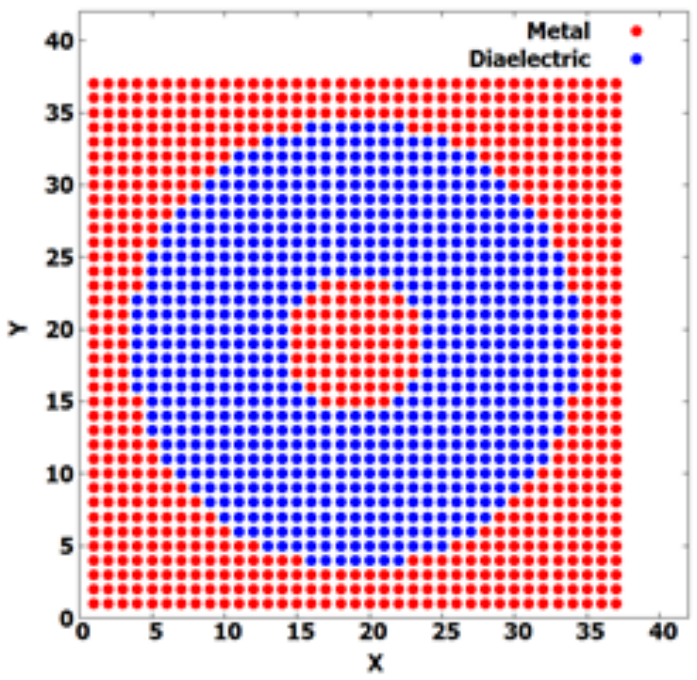

Fig. 3: Cross section of the cable’s grid system

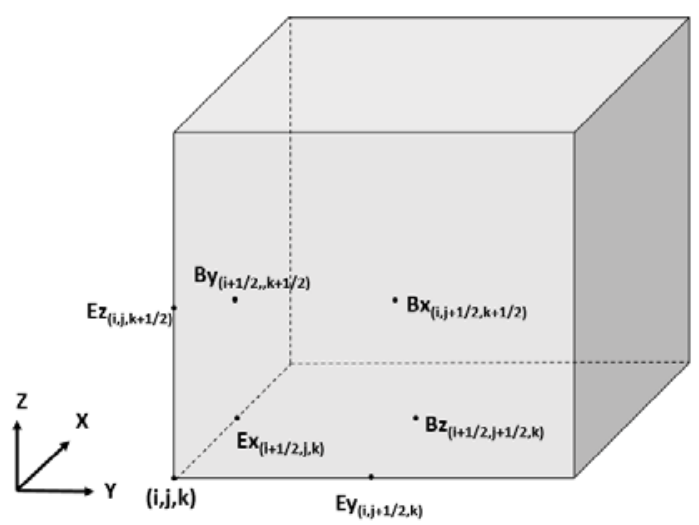

Fig. 4: a 3D FDTD grid with field positions

Based on the speed of light in the dielectric region and the time step chosen, the Courant-Friedrichs-Lewy condition is satisfied. The input position of the microwaves is set to $n_{z}=40$ where the electromagnetic field values are 
introduced in the $x y$ plane and are propagated in the right direction towards the end of the cable. The right end of the cable is set to have a closed boundary condition where the incoming waves are reflected backwards towards the other end. The left end of the cable is set to implement Mur's first order open boundary condition where the microwave leaves the cable. A Cartesian coordinate system is used in this model since it is the preferred coordinate system for FDTD. This causes some issues with simulating the geometry of the cable which is a perfect cylinder. Since the simulation model is limited to cubical grids, the simulated geometry ends up as an approximation which is illustrated in Figure 3. It can be seen that the model resembles a circle however it has shortcomings as well. The effects of these shortcoming will be discussed further in the following sections.

\section{Theory and Results}

\subsection{Electromagnetic Fields}

Since the three dimensional Cartesian model is used for this simulation, each grid is practically a perfect cube. One such grid along with the location of the field points is illustrated in Figure 4. Since none of the field points fits on the grid, developing a 3D FDTD simulation becomes complicated due to its calculation and indexing. Adjusting the original Maxwell equations to the simulation model in Figure 4 is a challenging task. Considering the basic scalar Maxwell equations in three dimensions adopted from ${ }^{13)}$ :

$$
\begin{aligned}
& \frac{\partial B_{x}}{\partial t}=\frac{\partial E_{y}}{\partial z}-\frac{\partial E_{z}}{\partial y} \\
& \varepsilon \frac{\partial E_{x}}{\partial t}=\frac{1}{\mu}\left(\frac{\partial B_{z}}{\partial y}-\frac{\partial B_{y}}{\partial z}\right)
\end{aligned}
$$

By transforming these equations to the grid point system in Figure 4, Maxwell's equations can be utilized in the simulation. Equations 3 and 4 indicate such transformation for $E_{x}$ and $B_{x}$ adopted from ${ }^{14)}$ :

$$
\begin{aligned}
& \varepsilon_{d i} \frac{E_{x\left(i+\frac{1}{2}, j, k\right)}^{n}-E_{x\left(i+\frac{1}{2}, j, k\right)}^{n-1}}{\Delta t}=\frac{1}{\mu_{0}}\left(\frac{B_{z\left(i+\frac{1}{2}, j+\frac{1}{2}, k\right)}^{n-\frac{1}{2}}-B_{z\left(i+\frac{1}{2}, j-\frac{1}{2}, k\right)}^{n-\frac{1}{2}}}{\Delta y}\right. \\
& \left.-\frac{\left.B_{y\left(i+\frac{1}{2}, j, k+\frac{1}{2}\right)}^{n-\frac{1}{2}}-B_{y\left(i+\frac{1}{2}, j, k-\frac{1}{2}\right)}^{n-\frac{1}{2}}\right)}{\Delta z}\right) \\
& \frac{B_{x\left(i, j+\frac{1}{2}, k+\frac{1}{2}\right)}^{n+\frac{1}{2}}-B_{x-\frac{1}{2}}^{\left.n-i, j+\frac{1}{2}, k+\frac{1}{2}\right)}}{\Delta t}=\frac{E_{y\left(i, j+\frac{1}{2}, k+1\right)}^{n}-E_{y\left(i, j+\frac{1}{2}, k\right)}^{n}}{\Delta z} \\
& -\frac{E_{z\left(i, j+1, k+\frac{1}{2}\right)}^{n}-E_{z\left(i, j, k+\frac{1}{2}\right)}^{n}}{\Delta y}
\end{aligned}
$$

And finally by adjusting the special indexing of the above equations and considering the leapfrog method for time step separation of $\mathrm{E}$ and $\mathrm{B}$ for the simulation code we will have:

$$
\begin{aligned}
E_{x(i, j, k)}=E_{x(i, j, k)}+ & \left(B_{z(i, j, k)}-B_{z(i, j-1, k)}\right) C_{y} \\
& -\left(B_{y(i, j, k)}-B_{y(i, j, k-1)}\right) C_{z} \\
B_{x(i, j, k)}=B_{x(i, j, k)}+ & \left(E_{y(i, j, k+1)}-E_{y(i, j, k)}\right) \frac{\Delta t}{\Delta z} \\
& -\left(E_{z(i, j+1, k)}-E_{z(i, j, k)}\right) \frac{\Delta t}{\Delta y}
\end{aligned}
$$

where $C_{y}=\frac{\Delta t}{\Delta y} \frac{1}{\varepsilon_{d i} \mu_{0}}$ and $C_{z}=\frac{\Delta t}{\Delta z} \frac{1}{\varepsilon_{d i} \mu_{0}}$.

\subsection{Microwave Input Method}

The microwave input power in a coaxial cable is calculated by ${ }^{10)}$ :

$$
\text { power }=\frac{2 \pi}{\sqrt{\frac{\mu_{0}}{\varepsilon_{d i}}}} v_{0 b}^{2} \ln \left(\frac{r_{\text {outer }}}{r_{\text {inner }}}\right)
$$

where $r_{\text {outer }}$ and $r_{\text {inner }}$ are the outer and inner radius of the cable respectively and the $v_{0 b}$ is the voltage between the cable's inner and outer layers. In the simulation, an adjustable parameter is multiplied to this equation for adjusting the input power value. This parameter does not affect the accuracy of the simulation in any way and is just a numerical simulation method. Therefore input field values are calculated by:

$$
B_{\theta}=v_{0 b} \frac{1}{r} \frac{1}{c_{D}} \sin (\omega t-\emptyset) \quad, \quad E_{r}=v_{0 b} \frac{1}{r} \sin (\omega t)
$$

where $C_{D}$ is the speed of light in the dielectric region and $\emptyset$ is the phase difference between the magnetic and electric fields. The field components $E_{r}$ and $B_{\theta}$ should be implemented on the cables cross section (Figure 3 ) and should be divided into their $x y$ components. In order to get the right $E \times B$ vector and the right values, therefore the following formulation is used:

$$
\begin{aligned}
& E_{x(i, j, k)}=E_{x(i, j, k)}+E_{r(i, j)} \frac{x}{r} \\
& E_{y(i, j, k)}=E_{y(i, j, k)}+E_{r(i, j)} \frac{y}{r} \\
& B_{x(i, j, k)}=B_{x(i, j, k)}-B_{\theta(i, j)} \frac{y}{r} \\
& B_{y(i, j, k)}=B_{y(i, j, k)}+B_{\theta(i, j)} \frac{x}{r}
\end{aligned}
$$

Applying Equations 9 to 12 would lead to the electric and magnetic field vectors at the input positions indicated in Figures 5 and 6 . The simulated waves are illustrated in Figures 7 through 12 . The figures indicates results for five wave period taken at a mid-plane cross section of the cable where the y values are constant. Since the wave in this simulation is supposed to act as a TEM wave the $E_{z}$ and $B_{z}$ values are expected to be close to zero. As it can be seen in Figure 9 the comparative value of $E_{z}$ with respect to the amplitude of $E_{x}$ wave in Figure 7 is too insignificant 


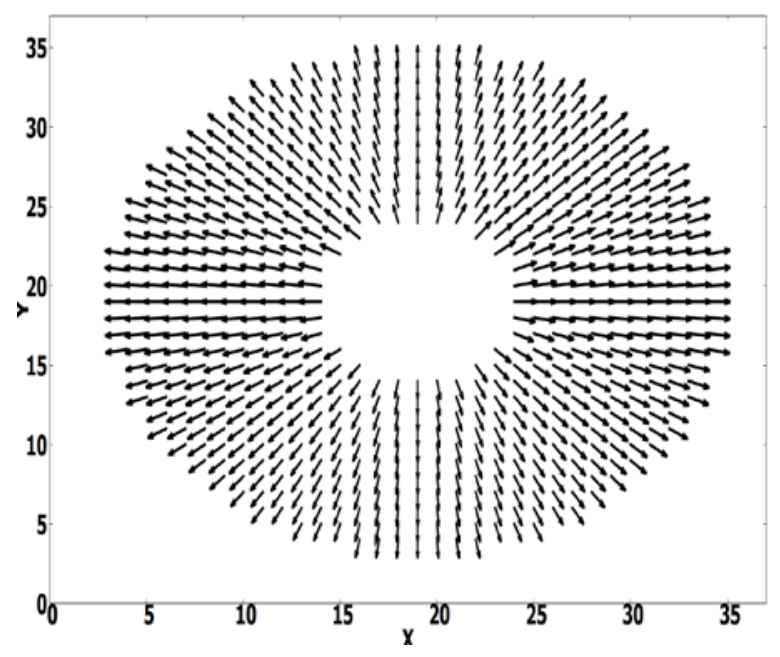

Fig. 5: Initial $E_{r}$ vectors at Microwave input plane

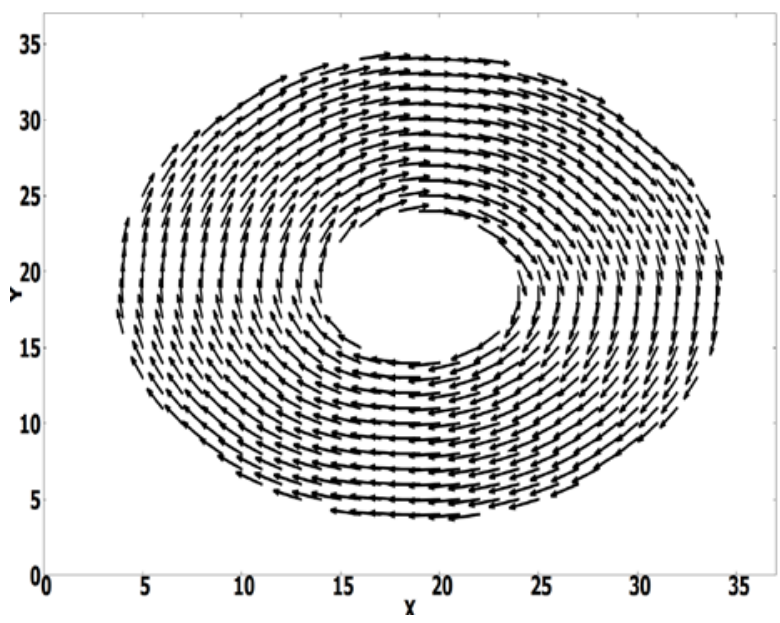

Fig. 6: Initial $B_{\theta}$ vectors at Microwave input plane

(24000 times smaller) which means that it can be ignored. Same thing applies to the magnetic field values depicted in Figures 11 and 12 . The $B_{z}$ value is half a million times smaller than $B_{y}$ which means it will not affect the accuracy of the simulation as well. Since this output data is taken at a plane in the center of the $y$ axis, $E_{y}$ and $B_{x}$ values corresponding to $E_{\theta}$ and $B_{r}$, are also expected to be zero. However as mentioned earlier since the simulated geometry of the cable is not a perfect cylinder we expect some imprecisions as well. According to Figures 8 and 11 since the amplitudes of $E_{y}$ and $B_{x}$ waves are four orders of magnitude smaller than their respective wave amplitudes, these imperfections can also be ignored as they will not cause any defects on the outcome of the simulation.

\subsection{Validation through Power Calculation}

In order to validate the results of the simulation, the power values are compared and inspected. To achieve that we will calculate the input power in two slices of the cable. One slightly ahead of the microwave input position (forward power) and one right before it (backward power). Equation 13 is used for calculating the input power.

$$
P_{\text {in }}=\frac{1}{\mu_{0}} \int_{A}(E \times B) \cdot d A
$$

By considering the input power in these two points, not only the power values can be checked but also the wave reflection can be seen as well. Since the input power was set to 10 Watts the input power fluctuations should be between 10 to minus 10 Watts as well. Furthermore we will calculate the output power which is the total power of the field values at each grid point and due to conservation of energy law, the input and output power values must be identical at all times during the simulation. Since this power is the results of changes of the electromagnetic fields over time as a result of our input wave it is called the output power. The output power (field power) is calculated as follows:

$$
P_{\text {out }}=\frac{\partial}{\partial t} U_{\text {field }}=\frac{\partial}{\partial t} \int_{V}\left[\frac{1}{2} \varepsilon E^{2}+\frac{1}{2 \mu} B^{2}\right] d V
$$

Through comparing the input power at both forward and backward positions with the field power (output power) values throughout the cable, the validity of the code can be examined. In order to investigate the power values, the simulation will consider the propagation of only two waves through the cable and the power fluctuations will be studied. Figure 13 shows power input fluctuations at forward position plotted over output power fluctuations for the two waves. The first upward fluctuations show the incoming waves whereas the second downward variations indicates the reflected waves. It should be noted that the amplitude of these waves varies from 0 to 20 Watts. This is because in Equation 13 the value of $\boldsymbol{E} \times \boldsymbol{B}$ is used to calculate the input power which for similar propagation direction maintains the same sign. This also means that for the waves on the right side of the figures the power value will be opposite since the propagation direction is reversed due to reflection. It is obvious in this figure that the input and output power values are identical throughout the duration of simulation which validates the accuracy of the wave propagation. Furthermore Figure 14 illustrates the average input power values throughout the simulation of two waves for the forward and backward position. The forward wave is the incident wave's average power which starts at the beginning of the simulation and reaches 10 Watts (the input power value) and goes back to zero once the waves have passed the forward calculation plane. Later the waves are reflected back from the closed boundary and reach the backward power calculation plane where the backward wave goes from 0 to -10 and back to 0 once the waves have passed the backward power calculation plane as well. This graph indicates the average power values of the incident and the reflected wave and since average power value of both waves is equal to the input power value, it validates the reflection process of the simulation. And finally Figure 15 shows the fluctuations of the power values indicated in Figure 14. It can be seen that the power fluctuations are similar for both calculation planes which also approves the simulation results. In order to avoid over plotting, only the incoming wave in the 


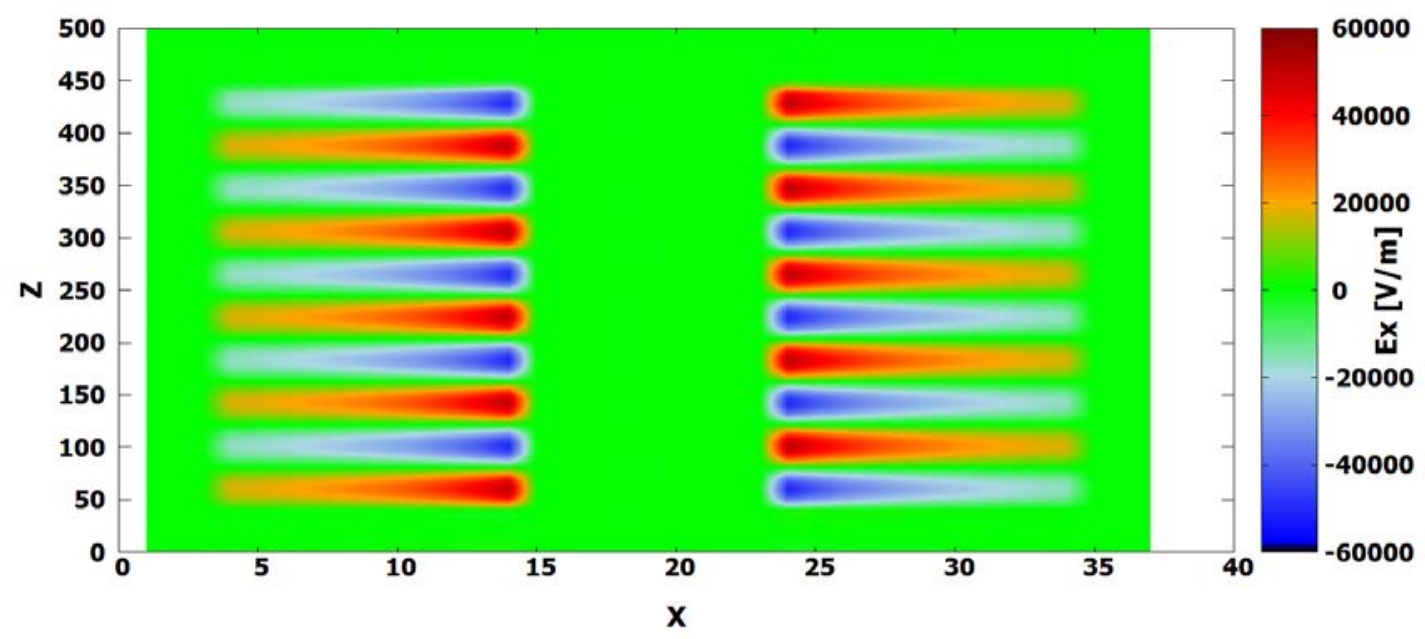

Fig. 7: $E_{x}$ field values through the coaxial cable

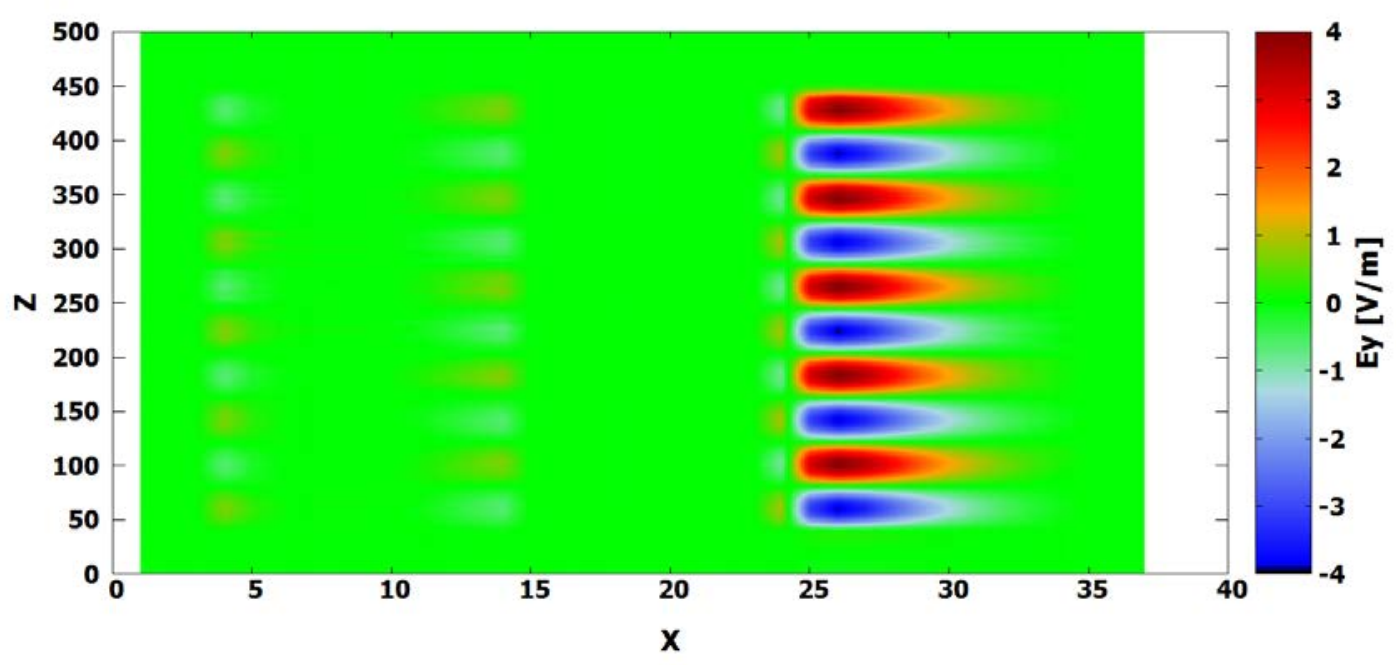

Fig. 8: $E_{y}$ field values through the coaxial cable

forward position and the reflected wave in the backward position are plotted.

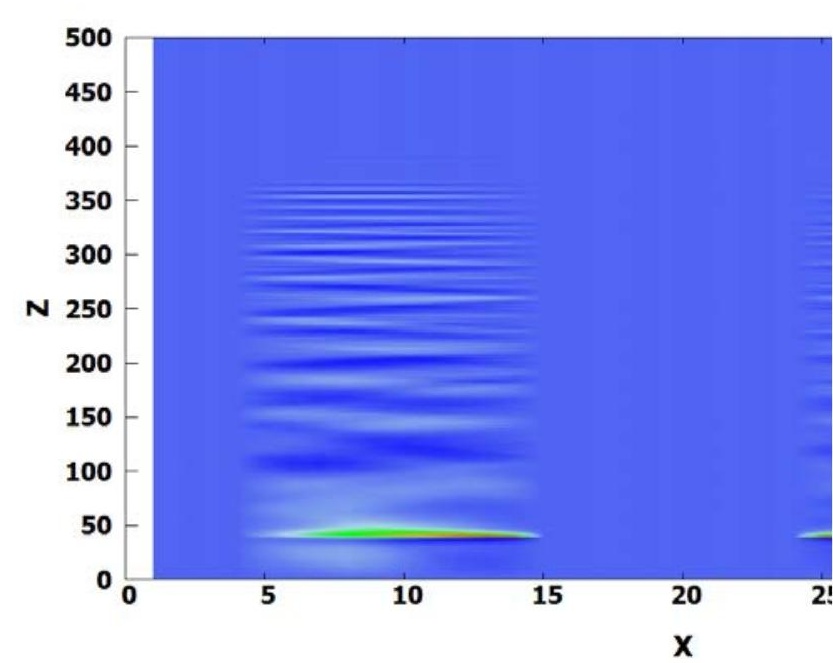

Fig. 9: $E_{z}$ field values through $\mathrm{t}$ 


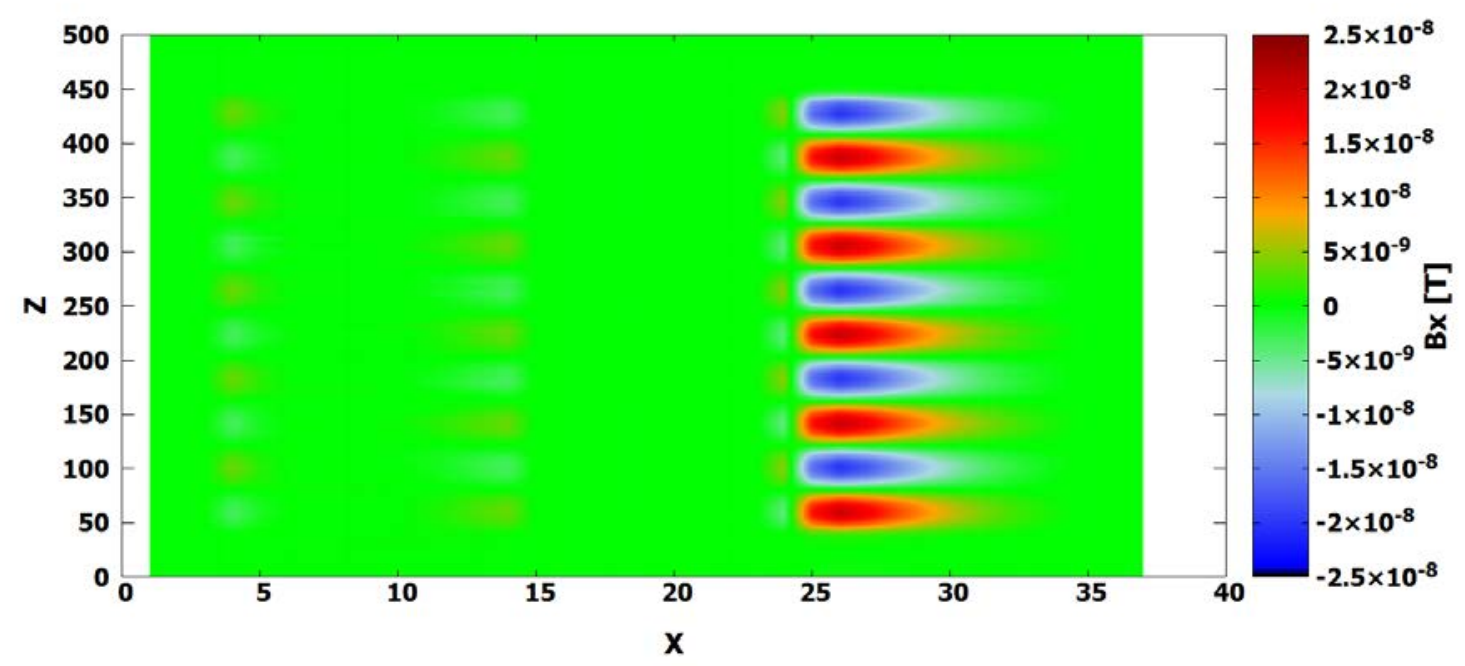

Fig. 10: $B_{x}$ field values through the coaxial cable

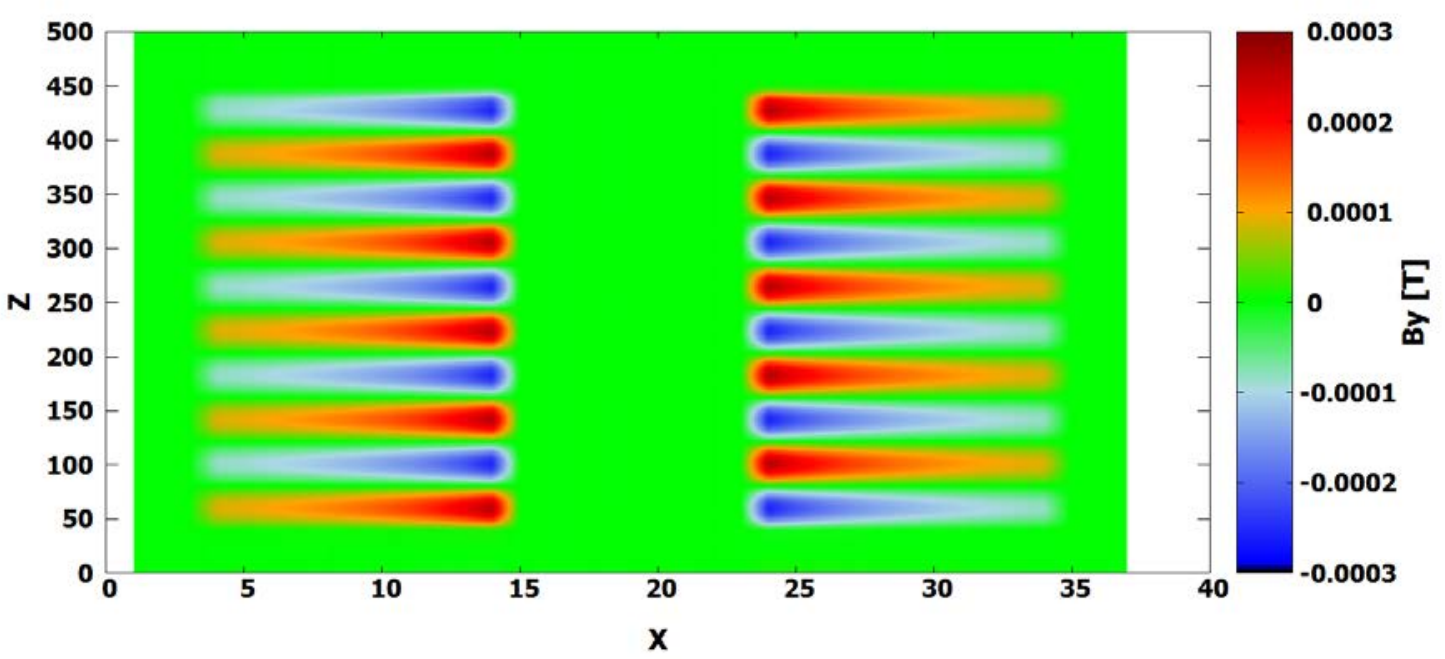

Fig.11: $B_{y}$ field values through the coaxial cable

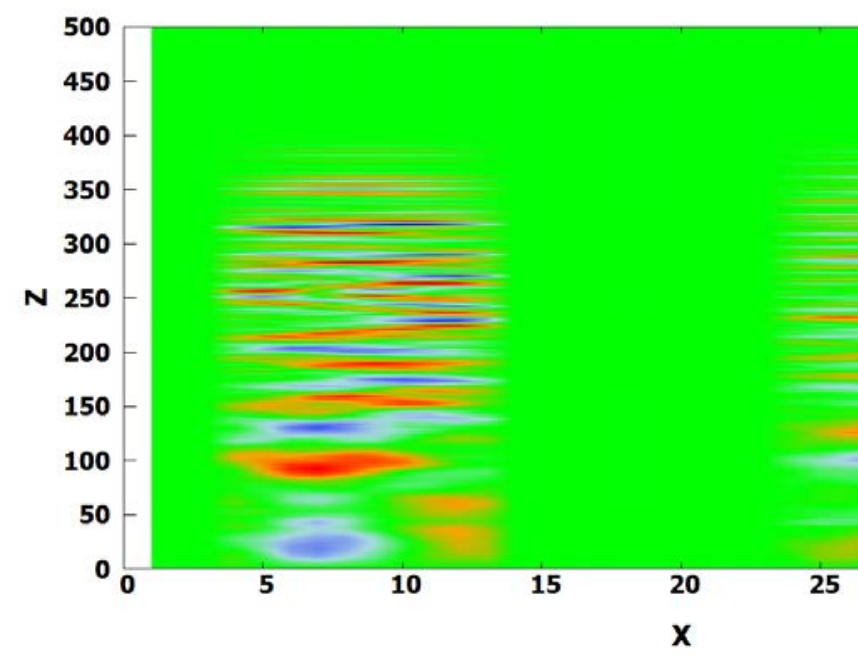

Fig. 12: $B_{z}$ field values through the 


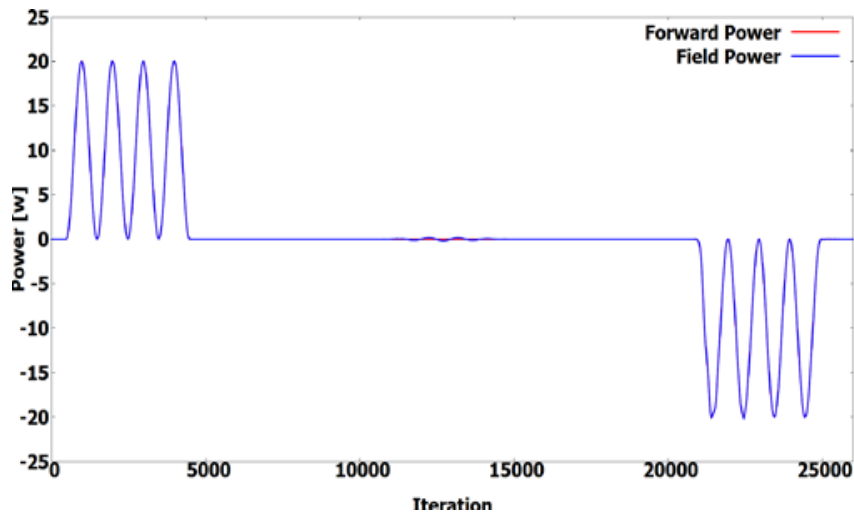

Fig. 13: Forward and Field power fluctuating values (Input and output Power) during simulation

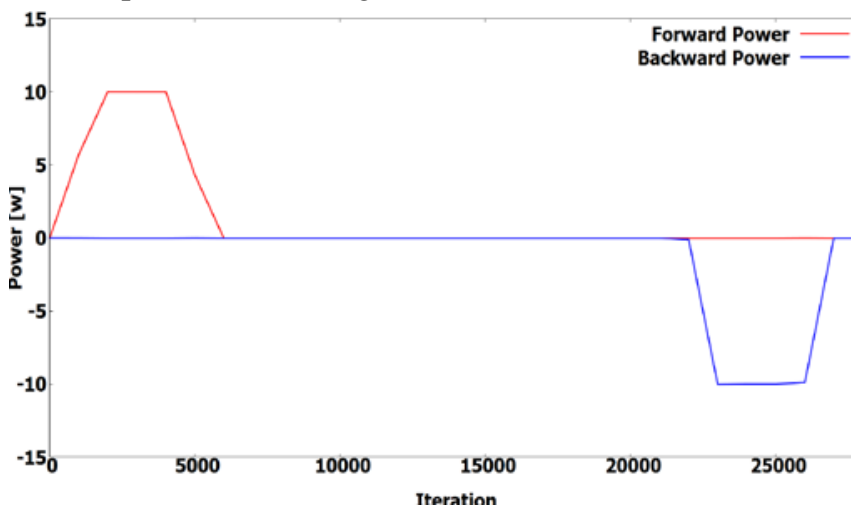

Fig. 14: Forward and backward power average values during simulation

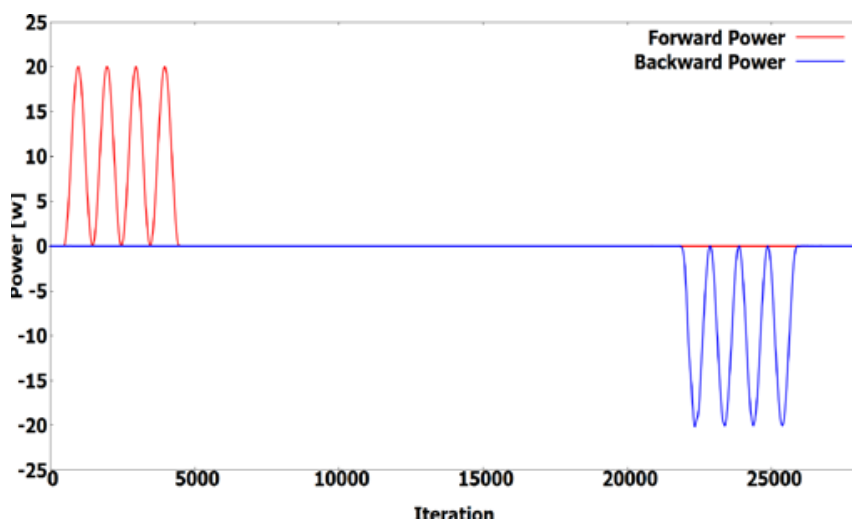

Fig. 15: Forward and backward power fluctuating values during simulation

\section{Comparison with Previous Methods}

The conventional method used previously in our research group for FDTD simulation adopted from references 4 to 6 had a contrasting microwave input method known as the four point method. In this method instead of having a plane where the microwave field values are introduced into the cable, four symmetric points around the central conductor rod are picked and only electric field value is implemented. Also the central conductor is reduced to one grid only which reduces the accuracy of this method even further. In this method the rest of the cable is ignored and only the field values in these four points are calculated. Since in our simulation we are considering the entire cable, we adjusted the four point input method for the entire input plane where the magnetic field input is ignored and the microwave is inputted in four perpendicular lines in input plane. We compared the simulation results of this method with the previously discussed simulation using exactly the same conditions as shown in previous outlines. The simulation results of the four point method are depicted in Figures 16 to 21. A simple side by side comparison of these and Figures 7 to 12 will clarify the improvements of the currently used simulation method. The first thing that is noticeable is that the waves in four point method are propagating in both directions from the input point. This is due to the fact that in order to achieve the correct pointing vector, the electric and magnetic field values of an entire plane should be considered and not just electric field values. Furthermore, by comparing the $E_{z}$ and $B_{z}$ graphs it is clear that the amplitude for the $E_{z}$ and $B_{z}$ waves in the four point method are larger than their counter parts in the plane method. This large value will result in further obvious inaccuracies illustrated in these figures. Further field ratio comparisons (simulation error) of the both methods are discussed in Table 1 for the original cylindrical coordinate system field values. Keep in mind that smaller field ratios are an indication of a more accurate simulation. Another advantage of the plane method can be seen that in figures 16 through 21 the waves are not symmetrical across the cable's cross section. This asymmetry is illustrated in figure 22 for the $E_{y}$ field values. This asymmetry would be resolved if the $E_{z}$ and $B_{z}$ values were manually set to zero. Another evident imprecision is in the $E_{y}$ and $B_{X}$ values which are corresponding to $\boldsymbol{E}_{\boldsymbol{\theta}}$ and $\boldsymbol{B}_{\boldsymbol{r}}$, that are supposed to be at least close to zero. It is clear in Figures 17 and 19 that the amplitude value of these two waves is considerably large in contrast to the new method. This large value is also caused by the $E_{z}$ and $B_{z}$ field values since manually setting them to zero will also resolve this issue as well. And finally the amplitude in the $E_{x}$ and $B_{y}$ graphs which are corresponding to $\boldsymbol{E}_{\boldsymbol{r}}$ and $\boldsymbol{B}_{\boldsymbol{\theta}}$, is half of what we expect in comparison to the new method. This is due to the fact that in four point method only the electric field are introduced and therefore the $\boldsymbol{E} \times \boldsymbol{B}$ vector is incorrect. This means that the wave in the conventional method propagates in both directions which leads to half the power being directed in the wrong course. This results in only half the power being propagated in the right direction.

Table 1. Field ratios (simulation error) evaluation for different input methods

\begin{tabular}{lcccc}
\hline Ratios / & $\frac{E_{\theta}}{E_{r}}$ & $\frac{B_{r}}{B_{\theta}}$ & $\frac{E_{z}}{E_{r}}$ & $\frac{B_{z}}{B_{\theta}}$ \\
\hline Plane & 0.00006 & 0.00083 & 0.00004 & 0.000002 \\
4-point & 0.0667 & 0.0667 & 0.00067 & 0.000267
\end{tabular}




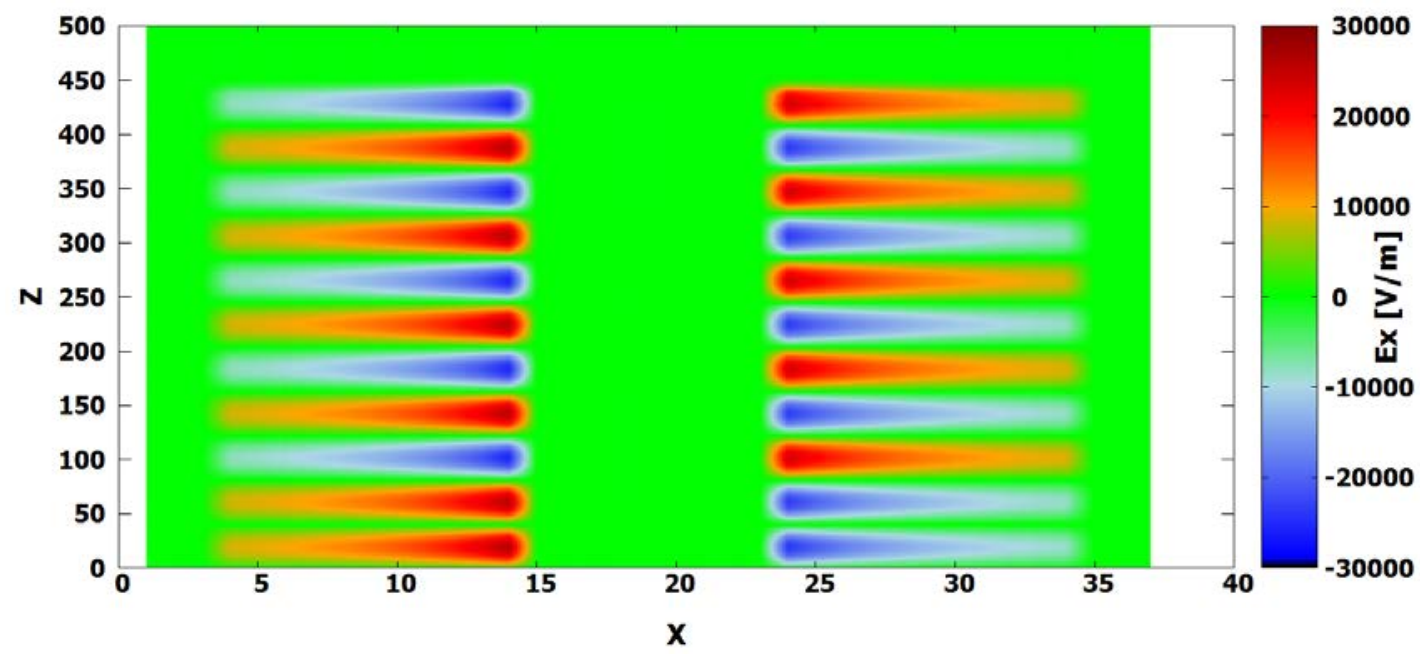

Fig. 16: $E_{x}$ field values through the coaxial cable in four point method

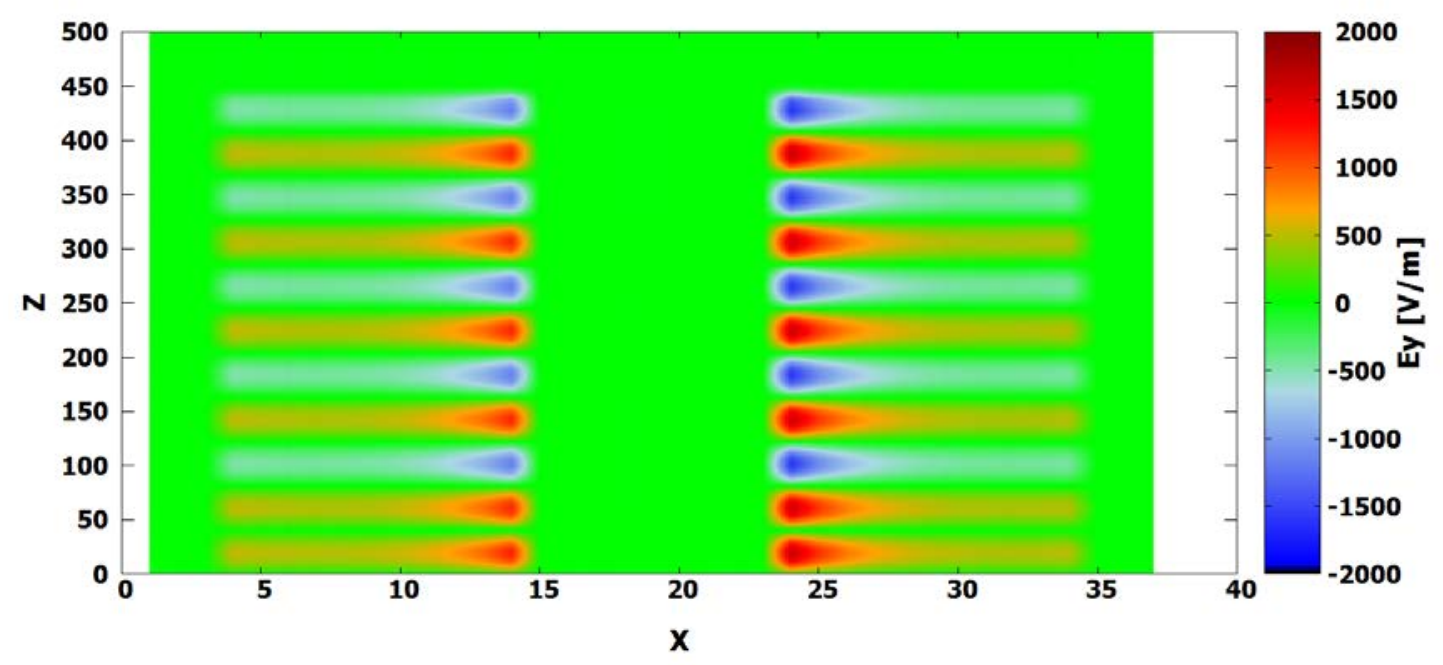

Fig. 17: $E_{y}$ field values through the coaxial cable in four point method

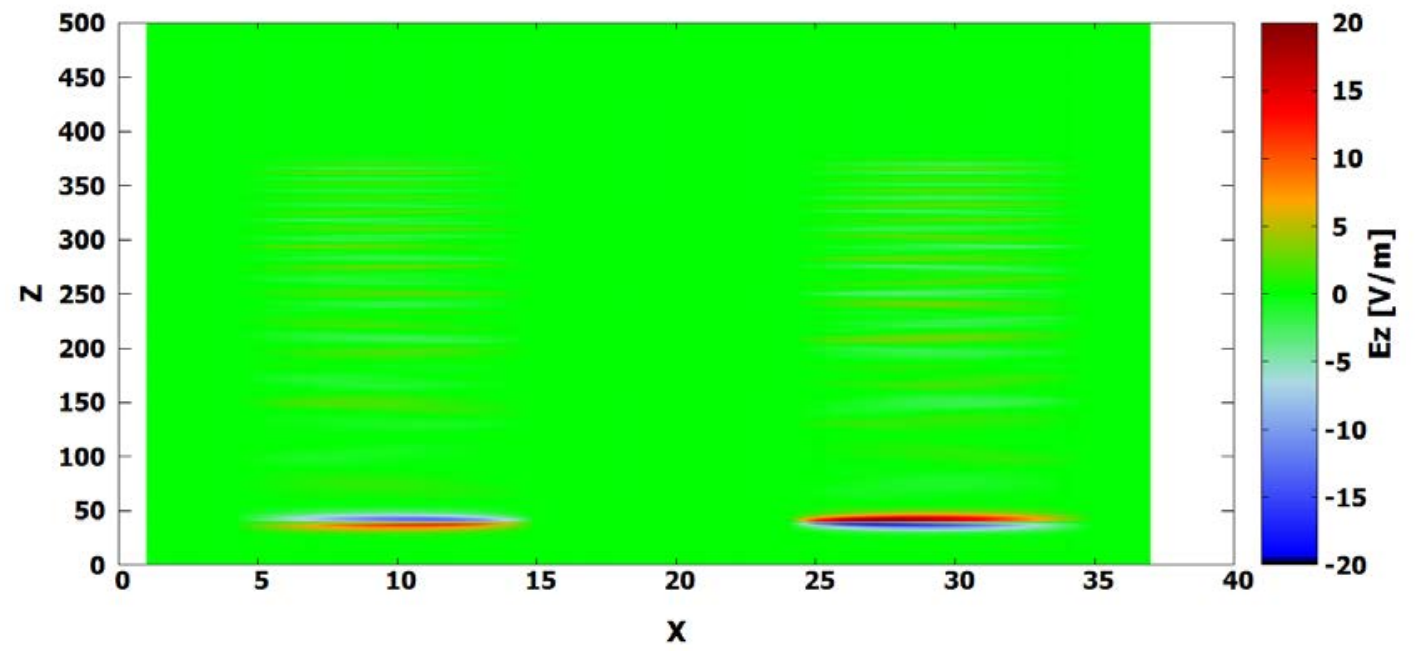

Fig. 18: $E_{z}$ field values through the coaxial cable in four point method 


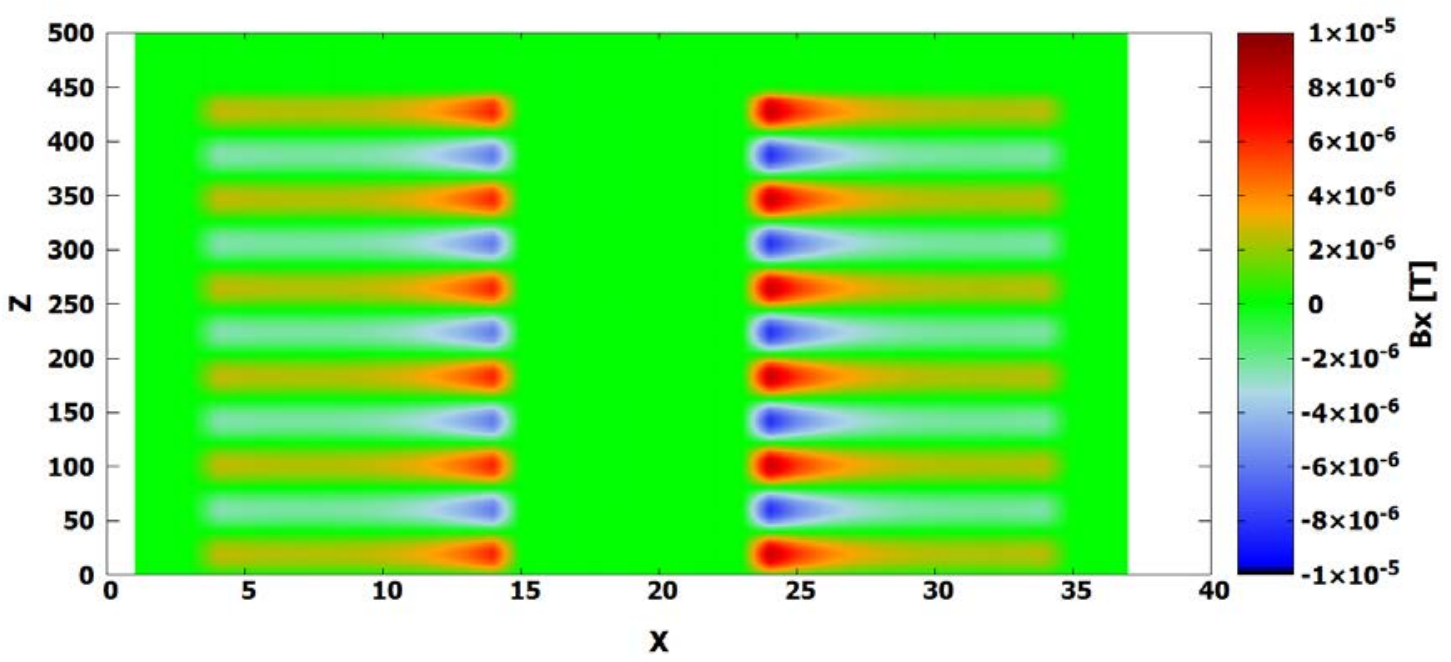

Fig. 19: $B_{x}$ field values through the coaxial cable in four point method

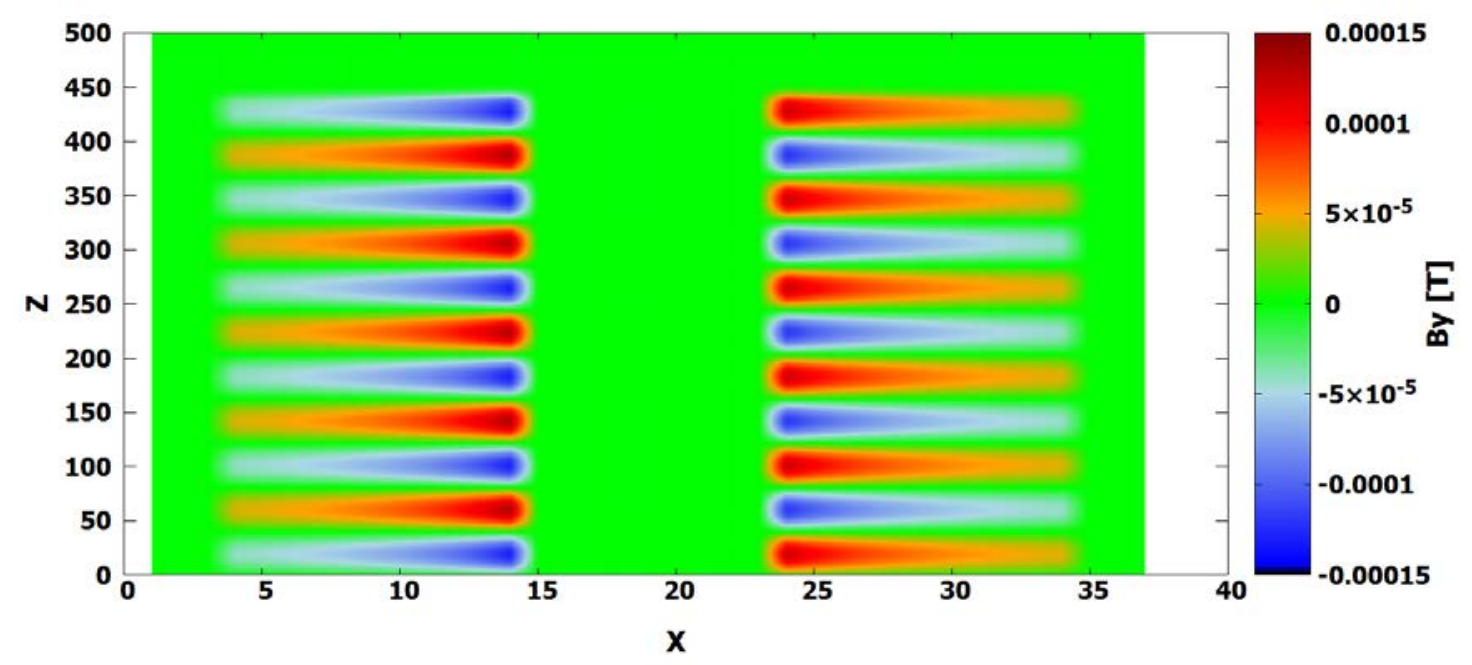

Fig. 20: $B_{y}$ field values through the coaxial cable in four point method

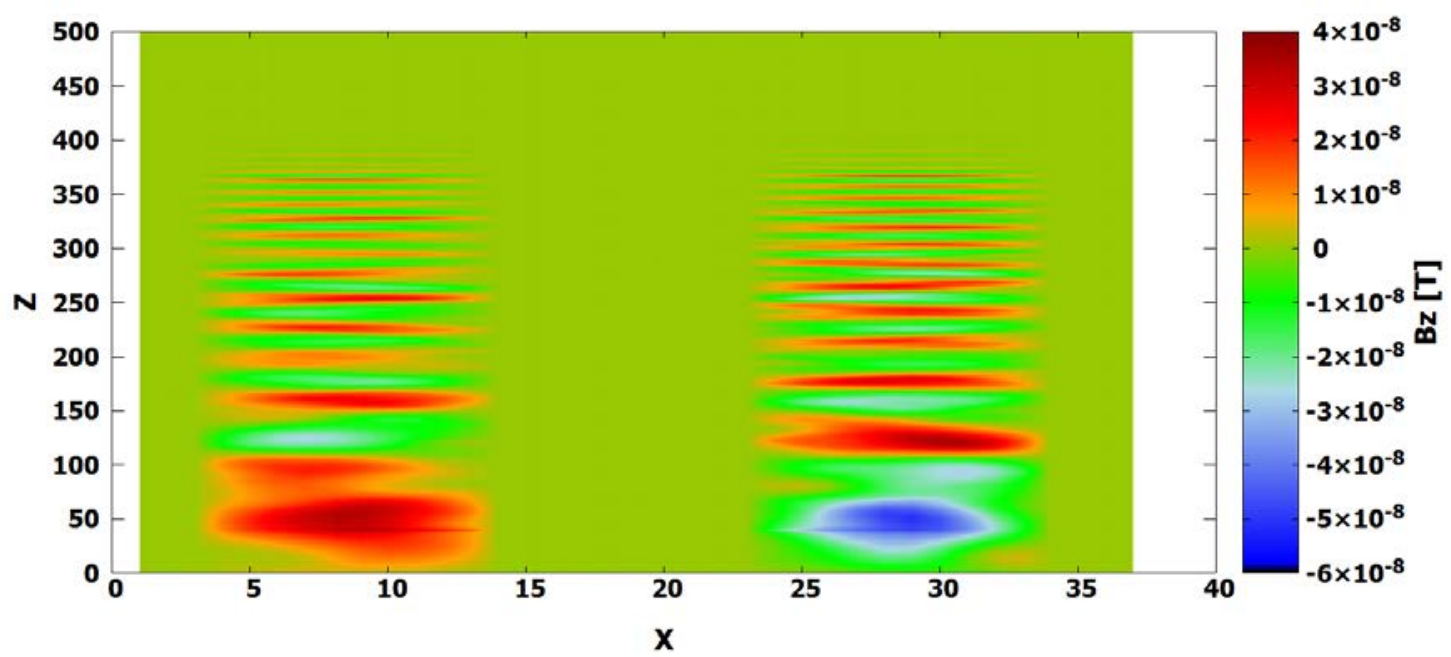

Fig. 21: $B_{z}$ field values through the coaxial cable in four point method 


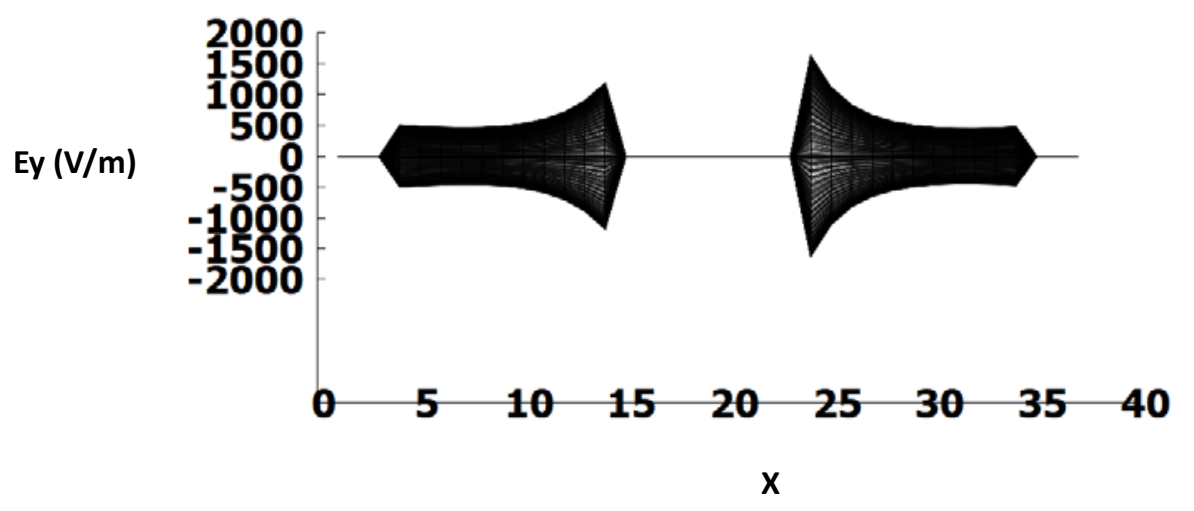

Fig. 22: Asymmetry in $E_{y}$ field values through the coaxial cable in four point method

\section{Conclusion}

The simulation for a microwave propagation inside a coaxial cable was discussed using FDTD method in a 3D model. The simulation results were discussed and their validation was investigated and a comparison with the previously utilized model was considered. The new model showed a highly accurate and realistic input of microwaves in a coaxial cable which will be utilized in future works for a more realistic simulation of an ion thruster. According to Table 1, the field ratios (simulation error) obtained by the conventional four point method are too large which leads to an inaccurate simulation of the microwaves. The plane method on the other hand offers a more accurate simulation and an acceptable field ratios which makes it a relatively perfect method for 3D simulation. This new model will replace the previous inaccurate four point method for future simulations in our research group and this in turn will result in development of better performing engines in future.

\section{Acknowledgements}

This research was supported by Green Asia Education Center, Kyushu University. We thank our colleagues from Takao Research Group (Yokohama National University) who provided insight and expertise that greatly assisted this research.

\section{References}

1) M. Hirakawa and M. Nakakita, "Simulation of Electron Cyclotron Resonance in a Microwave Discharge Ion Thruster", Trans. Japan Aero. Space Sci. Vol.47, (1999), pp.267-271, (in Japanese).
2) M. Shintani , "Numerical Analysis of Microwave Discharge Type Compact Ion Engine”, Masters Thesis. Kyushu University Fukuoka, Japan, (2010).

3) H. Masui, T. Tanoue, H. Nakashima and I. Funaki, "Development of an Electromagnetic Particle Code for Space Propulsion Application Using ECR Discharge”, 24th International Symposium on Space Technology and Science, paper ISTS-b-11, (2004).

4) T. Kanagawa, N. Yamamoto, Y. Kajimura and H. Nakashima, "Numerical Simulation of Internal Plasma in a Miniature Microwave Discharge Ion Thruster" IEPC-2007-190, (2007).

5) M. Kusuda, Y. Sasagawa, N. Yamamoto and H. Nakashima, "Numerical Simulation Analysis of Microwave Discharge Neutralizer Internal Physics" STEP-2013-011, (in Japanese).

6) M. Kusuda, Y. Sasagawa, N. Yamamoto and H. Nakashima, "Two-Dimensional Numerical Analysis of a Miniature Microwave Discharge Neutralizer" JSASS-2011-S026, (in Japanese).

7) H. Koizumi and H. Kuninaka, "Miniature Microwave Discharge Ion Thruster Driven by 1 Watt Microwave Power” J. Propul. \& Power, Vol 26, pp601-604, (2010).

8) H. Masui, U. Tashiro, N. Yamamoto, H. Nakashima, I. Funaki, "Analysis of Electron and Microwave Behaviour in Microwave Discharge Neutralizer" Trans Japan. Soc. Aero. Space. Sci. Vol 49, pp 87-93, (2005). 
9) K. Kubota, H. Watanabe, N. Yamamoto, H. Nakashima, T. Miyasaka, I. Funkai, “Three Dimensional HybridPIC Analysis on Electron Extraction of a Microwave Neutralizer" Proceedings of the Japan Aeronautics and Space Administration Vol 63, issue 5 , pp197-203, (2015).

10) M. Nakashima, Microwave Engineering Foundations and Principles, Morikita Electrical Engineering Series (3), (1995), pp 67-69.

11) H. Kousaka, K. Ono, "Numerical Analysis of the Electromagnetic Fields in a Microwave Plasma Source Excited by Azimuthally Surface Waves” Japan Journal of Physics. Vol 41, pp 2199-2206, (2002).

12) Y. Takao, H. Koizumi, K. Eriguchi, K. Komurasaki, and K. Ono, "Three-dimensional Particle in Cell Simulation of Miniature Plasma Source for a Microwave Discharge Ion Thruster”, Plasma Source Sci. Technol, Vol.23, pp 064004-064015 (2012).

13) F.Chen. Francis, in Introduction to Plasma Physics and Controlled Fusion, 2nd ed. by Plenum Publishing Corporation, (1974).

14) K. Yee, "Numerical Solution of Initial Boundary Value Problems Involving Maxwell's Equations in Isotropic Media” IEEE Trans, Vol 14, pp 802-307 (1966). 